\title{
A comparative analysis of different coding strategies on NMF-based codebooks from rat bulbar activity patterns
}

\author{
Johanna Degen*, Christiane Wegner, Amir Madany Mamlouk \\ From 1st International Workshop on Odor Spaces \\ Hannover, Germany. 4-7 September 2013
}

One object of olfactory research is to understand the coding map of an olfactory bulb. Even after decades of olfactory research, it is still not possible to predict if two odorants smell similar or not without asking human subjects and/or using prior knowledge on exactly these substances. There is only a single source of data, which includes the activity of the whole bulb under exposure of a single compound: The uptake data from Leon \& Johnson [1]. Given that the activity is a chemotopic ordered multifactorial code, there are several possible ways these codes might be implemented by nature. So far, specific bulb regions were declared as modules by using the Nonnegative Matrix Factorization. To examine the codes we imagine the modules as letters of an alphabet. Analog to the orthography and grammar of a language, we want to find rules that explain the meaning of the composition of individual words. We consider an alphabet consisting of nine letters, one for each module. Odorants are characterized by module activities and thus form words of the alphabet. The number of activated modules classifies the odorants into groups with different word lengths. To understand complex words, an intuitive approach is to first concentrate on the relations between the letters to each other. We present three different models that describe how odor quality on this modular abstraction could be measured.

First of all there is a chemotopic approach, which assumes that odorants with similar chemical epitopes address the same types of receptors. But the corresponding glomeruli are not necessarily close to each other. In order to implement this model we define regions of interest using above-threshold uptake induced by incoming receptor neuron activity. The second model combines the chemotopic approach with the spatial arrangement of the glomeruli. Accordingly the similarity of odorants is shown in the activity of nerve tracts of neighboring glomeruli. We found a suitable measure for this model in the so called Earth Mover's Distance [2]. The last model relates to a hierarchical decomposition of the bulb into 4, 6, and 9 modules, which was found during successive increase of the number of NMF vectors. Based on this "data genealogy", we used a slightly modified version of the dice coefficient to compare the similarity of the given 9 modules, the letters of our alphabet, in this context. Finally, we will present a preliminary comparison between these models and a psychophysical study that measured perceived similarity of odorants chosen in the context of this setup.

Published: 16 April 2014

\section{References}

1. Johnson BA, Leon M: Chemotopic odorant coding in a mammalian olfactory system. J Comp Neurol 2007, 503:1-34.

2. Rubner $Y$, Tomasi $C$, Guibas LJ: A metric for distributions with applications to image databases. In Sixth Int Conf Comput Vis (IEEE Cat No98CH36271) Narosa Publishing House; 1998, 59-66.

doi:10.1186/2044-7248-3-S1-P5

Cite this article as: Degen et al:: A comparative analysis of different coding strategies on NMF-based codebooks from rat bulbar activity patterns. Flavour 2014 3(Suppl 1):P5. 